University of Nebraska - Lincoln

DigitalCommons@University of Nebraska - Lincoln

4-10-2011

\title{
The North American Drought Monitor: A Continental Approach
}

Mark Svoboda

University of Nebraska - Lincoln, msvoboda2@unl.edu

Follow this and additional works at: https://digitalcommons.unl.edu/natrespapers

Part of the Natural Resources and Conservation Commons

Svoboda, Mark, "The North American Drought Monitor: A Continental Approach" (2011). Papers in Natural Resources. 358.

https://digitalcommons.unl.edu/natrespapers/358

This Article is brought to you for free and open access by the Natural Resources, School of at DigitalCommons@University of Nebraska - Lincoln. It has been accepted for inclusion in Papers in Natural Resources by an authorized administrator of DigitalCommons@University of Nebraska - Lincoln. 


\section{The North American Drought Monitor:}

\section{A Continental Approach}

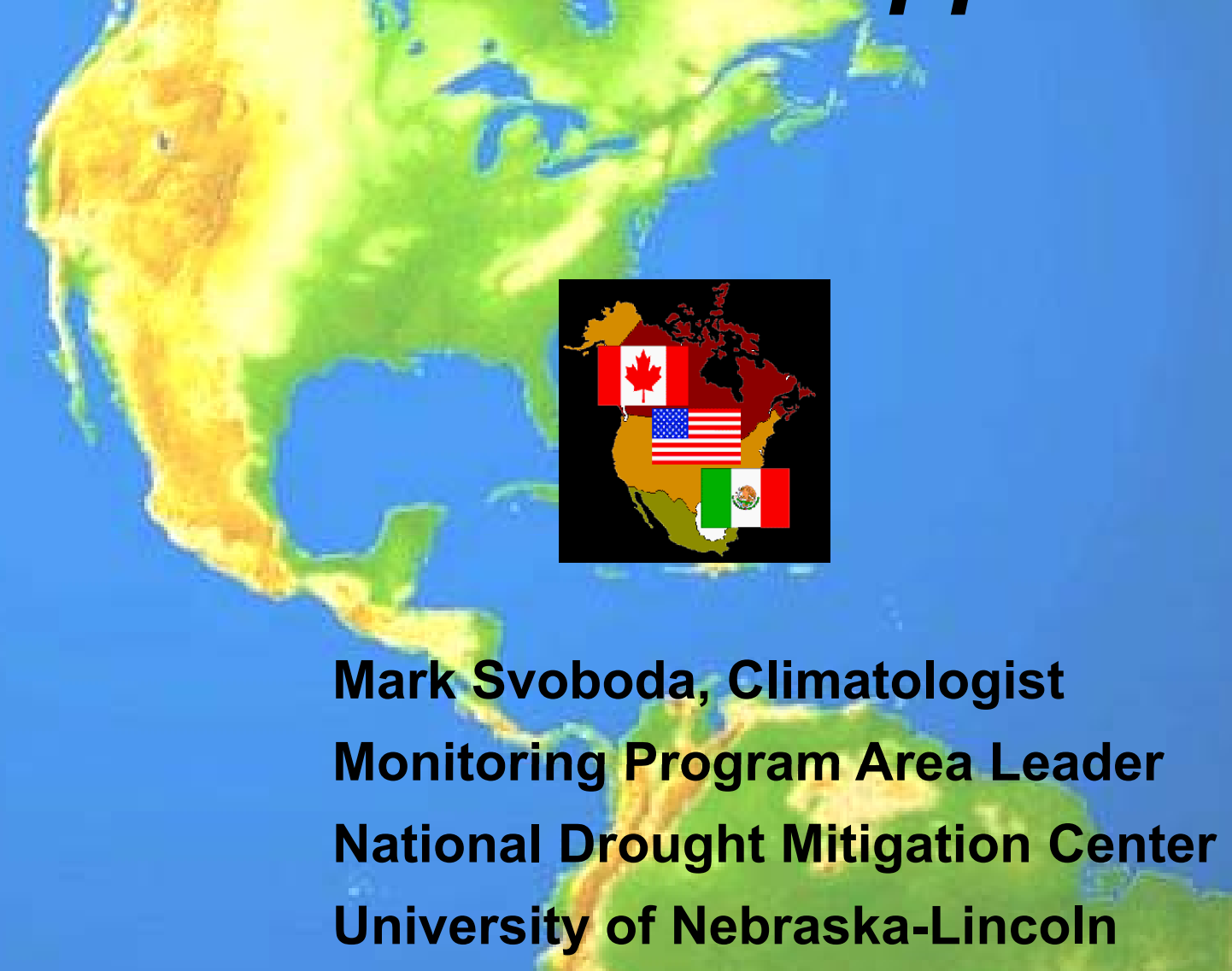

GEOSS Drought Workshop XL: Monitoring through Earth Observations Sydney, Australia, April 10, 2011 


\section{The U.S. Drought Monitor}

Since 1999, NOAA (CPC and NCDC), USDA, and the NDMC have produced a weekly composite drought map -- the U.S. Drought Monitor -- with input from numerous federal and non-federal partners

August 3, 1999

Experimental U.S. Drought Monitor

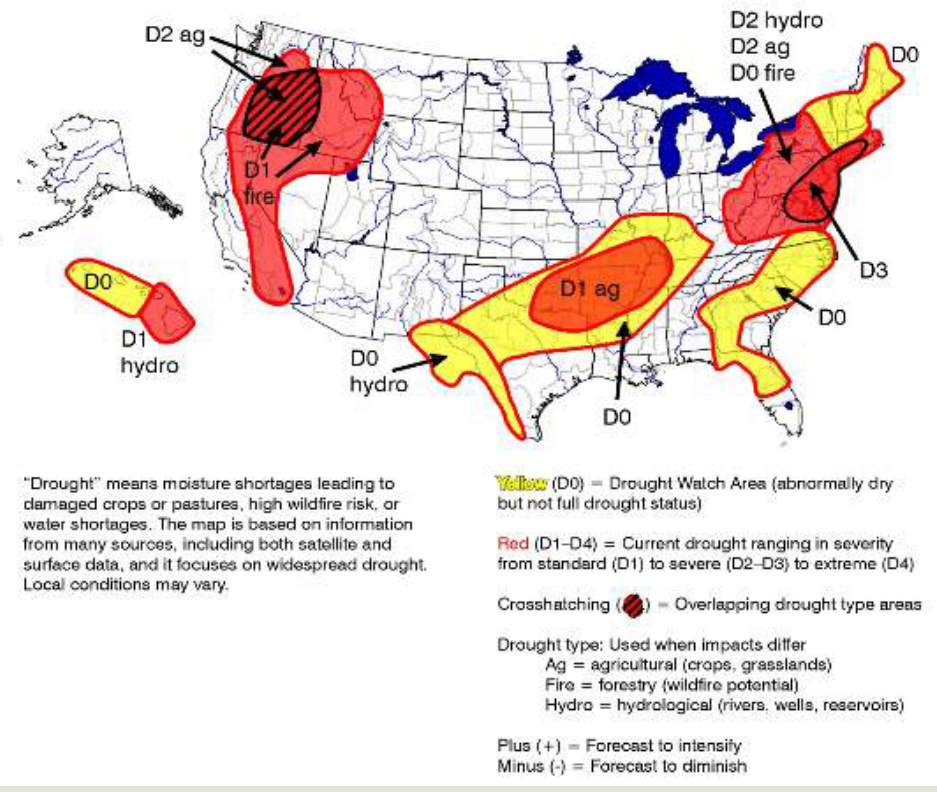

U.S. Drought Monitor April 16, 2002

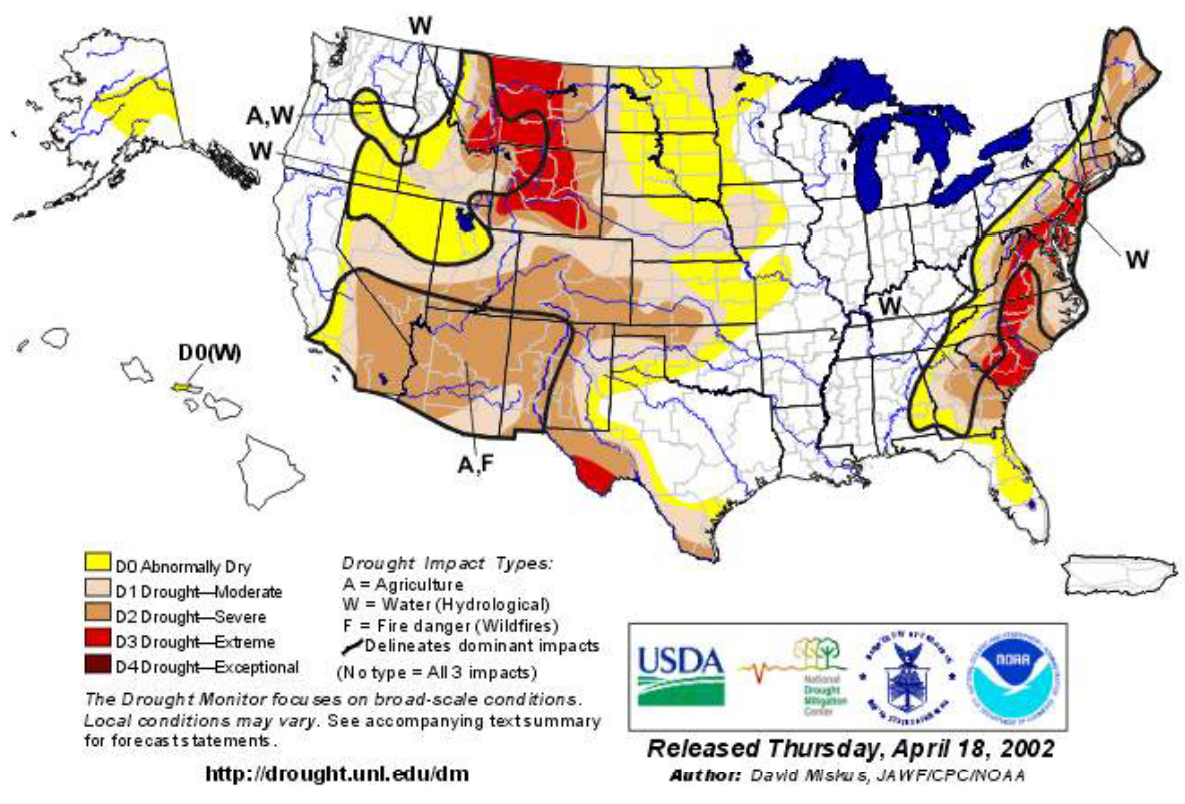




\section{History of the North American Drought Monitor (NADM)}

$>$ The concept for the NADM was developed and discussed in 2002

$>$ The first NADM map was released in March 2003

$>$ The first NADM map in all three languages (English, Spanish, and French)was released in October 2003

Nebraskia 


\section{History of the NADM}

$>$ The United States started off as the only country to author and organize the map

$>$ Canada began authoring in January 2006

$>$ Mexico began authoring in October 2008

Nebraska 


\section{North American Drought Monitor Partners}

\section{$>$ Canada}

- Agriculture and Agrifood Canada

- Environment Canada

- Meteorological Service of Canada

$>$ Mexico

- National Meteorological Service of Mexico (SMN-Servicio Meteorologico Nacional)

- CONAGUA (Comision Nacional del Agua)

$>$ United States

- National Drought Mitigation Center

- National Climatic Data Center

- Climate Prediction Center

- United States Department of Agriculture

Nebraska

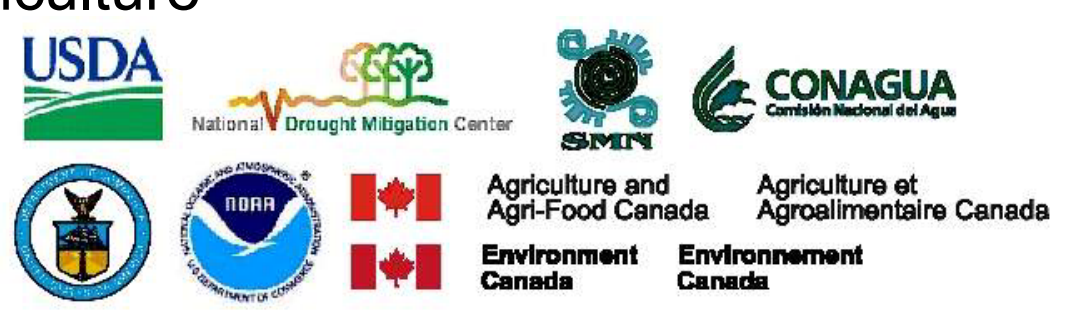




\section{North American Drought Monitor}

February 28, 2011

Released: Friday March 18, 2011

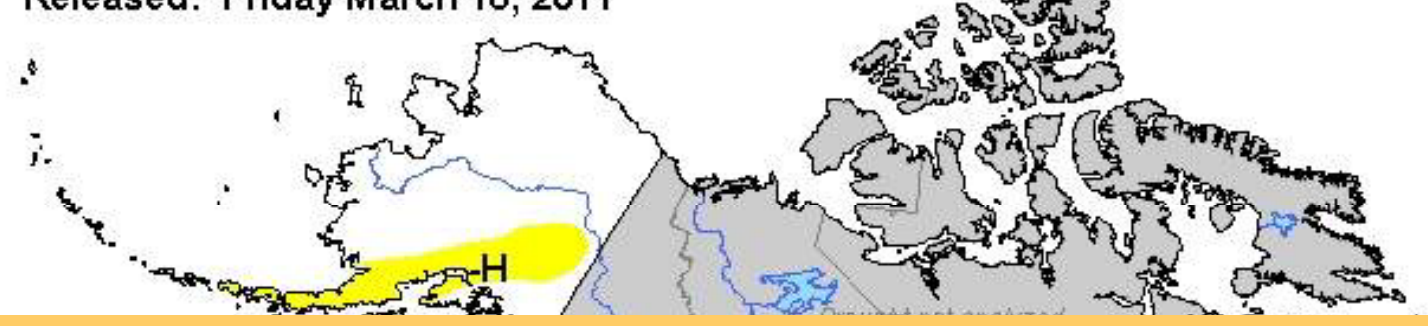

Analysts:

Canada - Trevor Hadwen Dwayne Chobanik Richard Rieger

Mexico - Fernando Romero Reynaldo Pascual Adelina Albanil

U.S.A. - Mark Svoboda*

Laura Edwards

\section{Hon OW \\ D4 Drought - Exceptional}

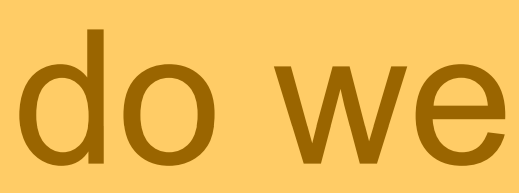

get there

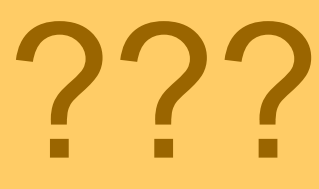

Drought impact Types:

$\sim$ Delineates dominant impacts

$\mathrm{A}=$ Agriculture

$\mathrm{H}=$ Hydrological (Water)
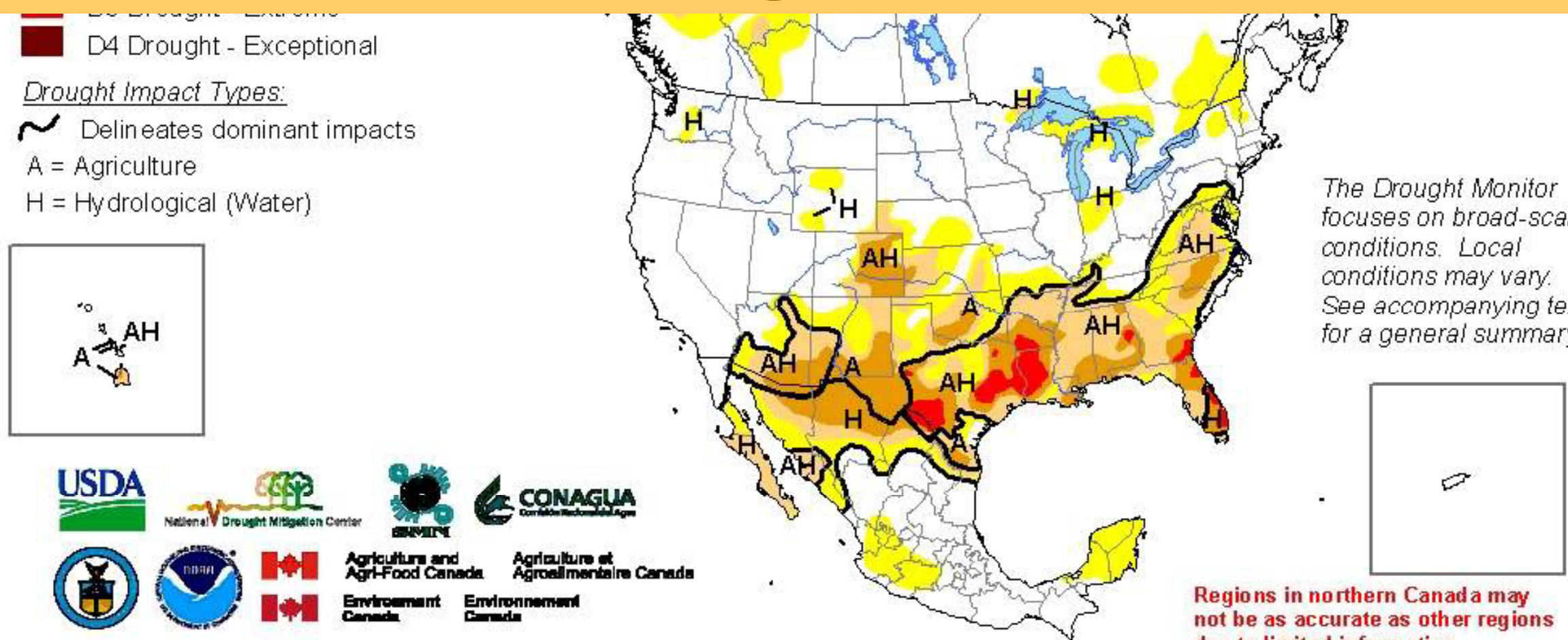

Regions in northern Canada may not be as accurate as other regions due to limited information. 


\section{Percentile Ranking Approach}

U.S. Drought Monitor September 1 2009

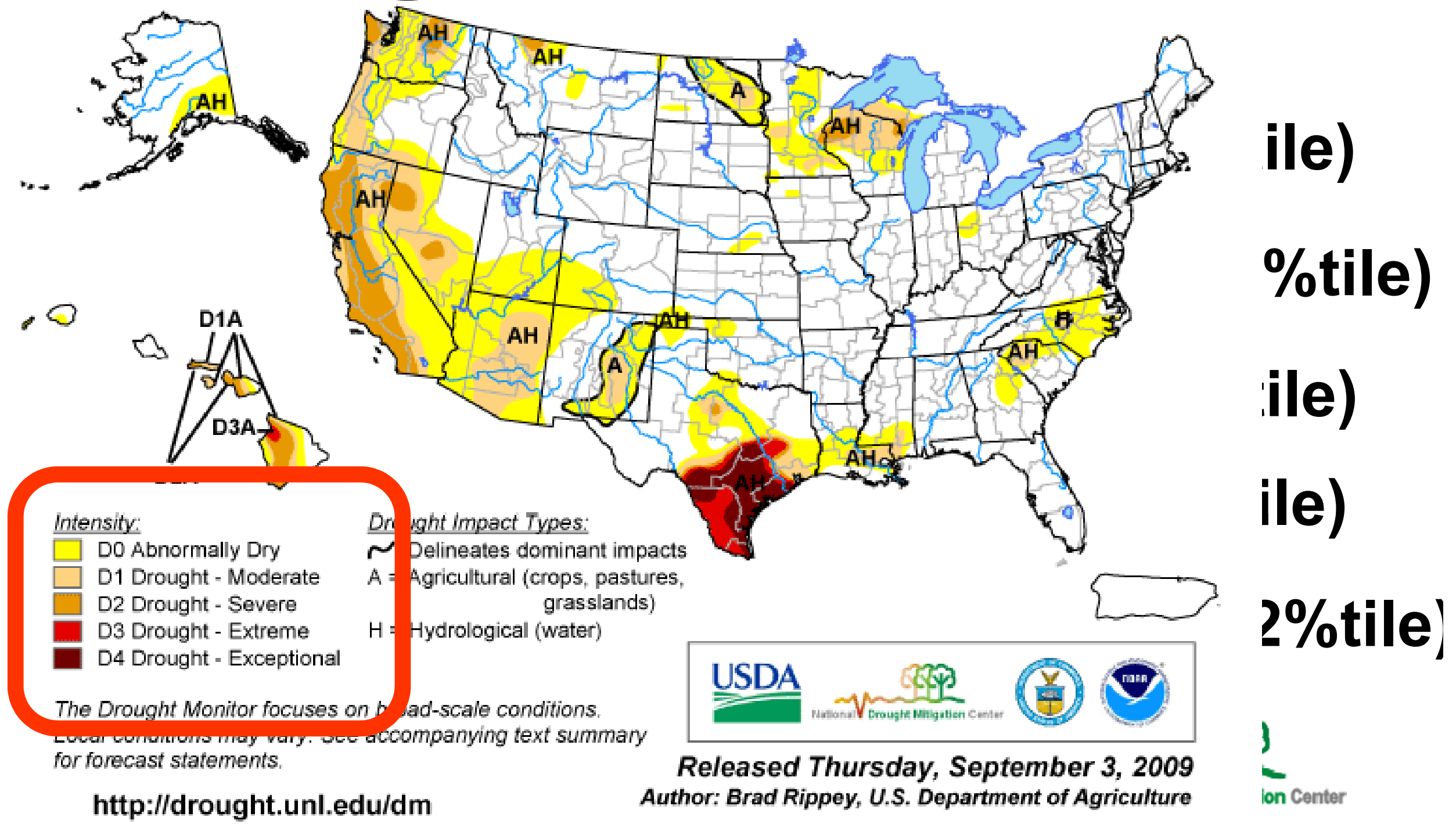




\section{U.S. Drought Monitor}

March 1, 2011

Valid 7 a.m. EST
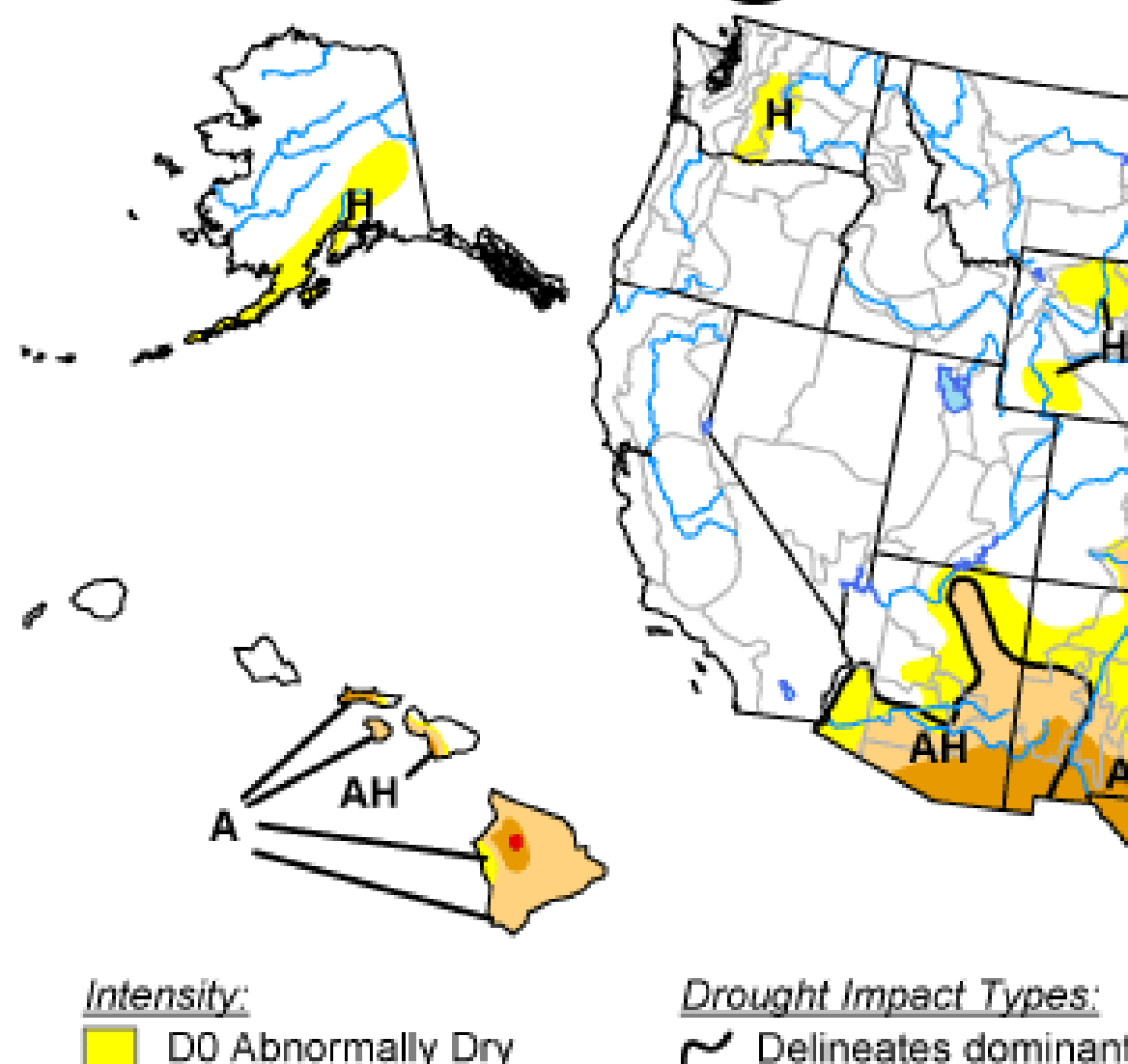

Intensity:

$\square$ D0 Abnormally Dry

D1 Drought - Moderate

D2 Drought - Severe

D3 Drought - Extreme

$\sim$ Delineates dominant impacts

$\mathrm{A}=$ Agricultural (crops, pastures,

grasslands)

$\mathrm{H}=$ Hydrological (water)

D4 Drought - Exceptional

The Drought Monitor focuses on broad-scale conditions. Local conditions may vary. See accompanying text summary for forecast statements.

http://drought.unl.edu/dm

Released Thursday, March 3, 2011 Author: Laura Edwards, Western Regional Climate Center 


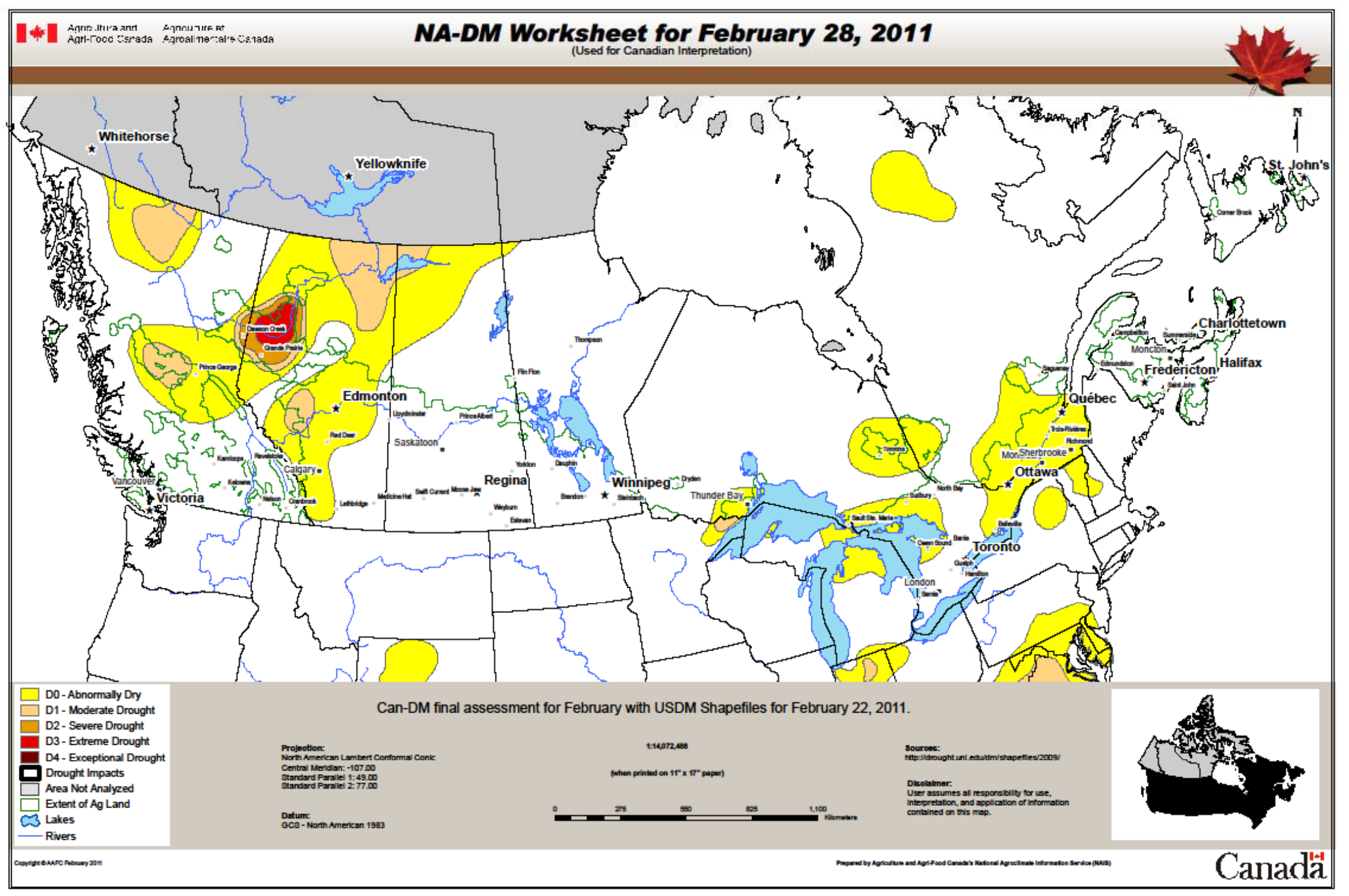

\section{Nebraska}




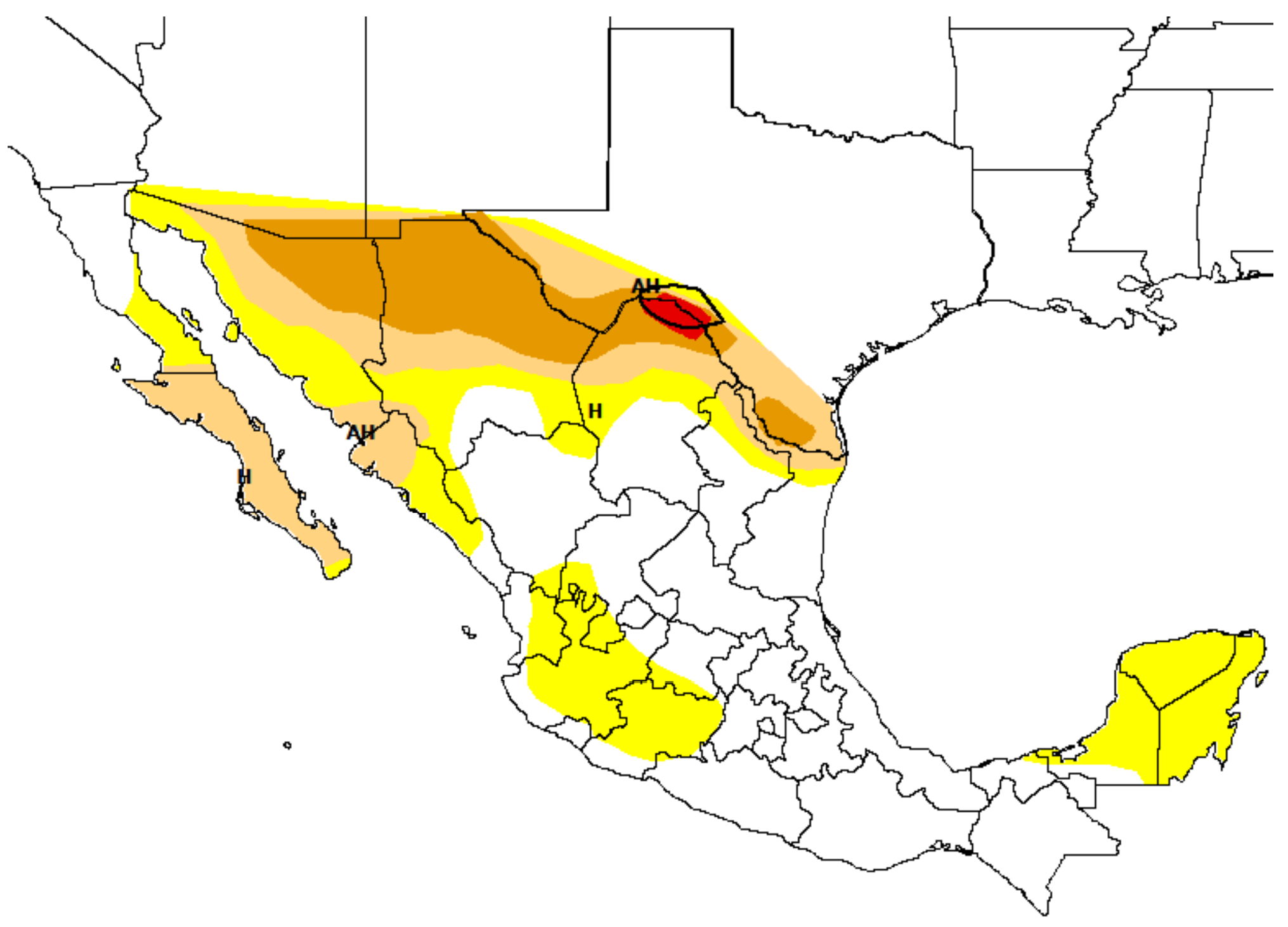

Nebraskia Lincoln 


\section{North American Drought Monitor}

February 28, 2011

Released: Friday March 18, 2011

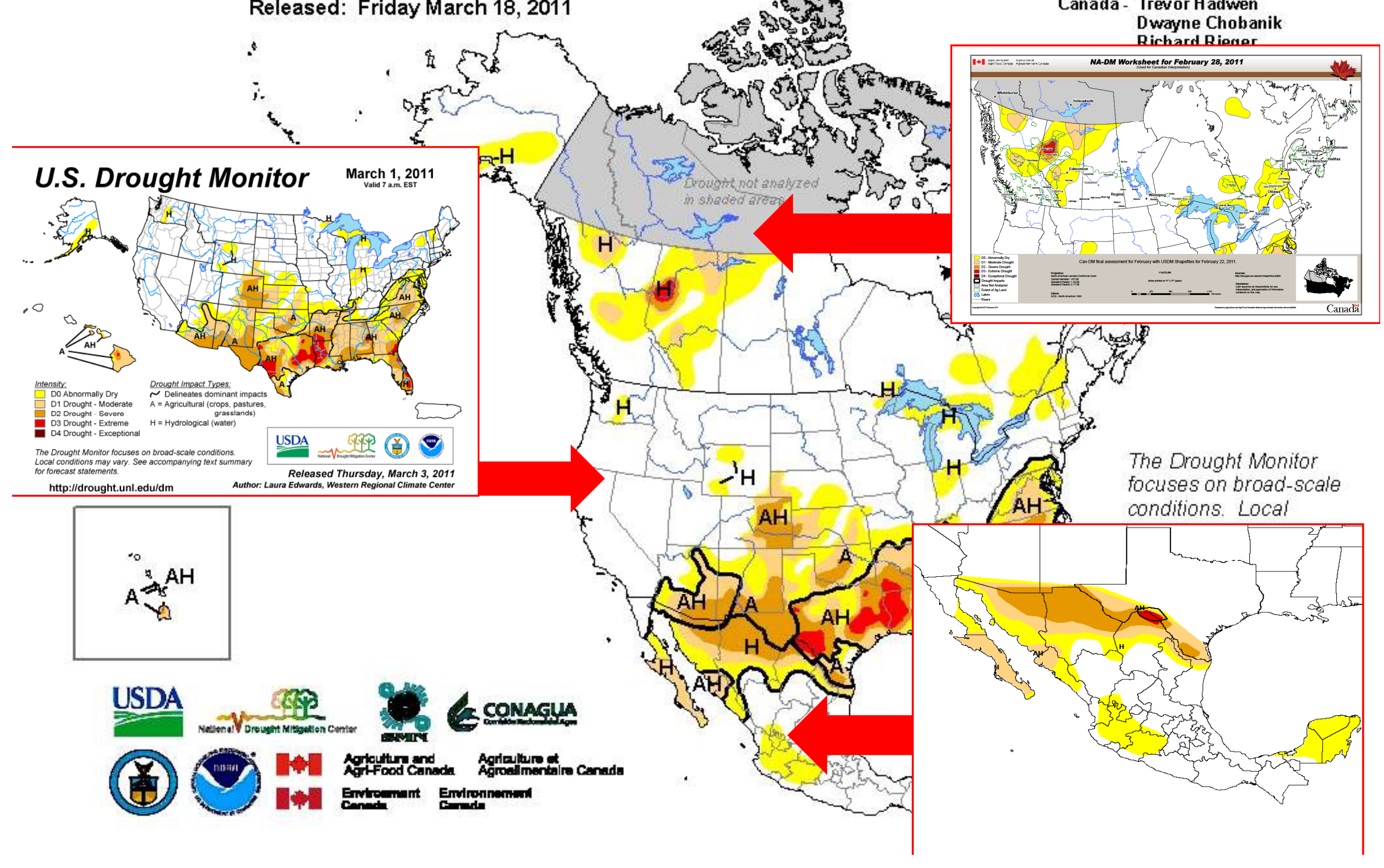




\section{All 3 Countries merged together using ArcGIS shapefiles}

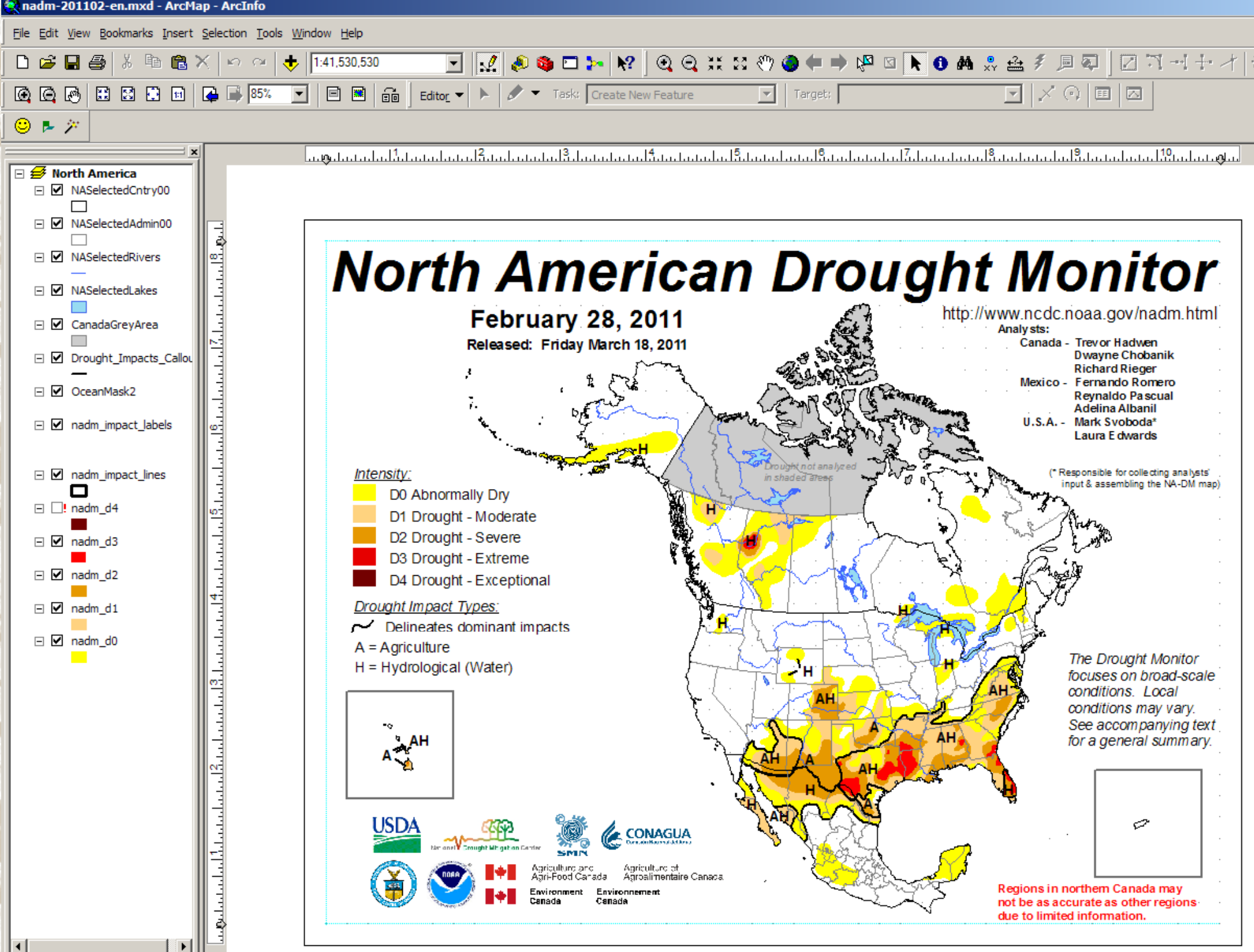


NOAA Satellite and Information Service

National Environmental Satellite, Data, and Information Service (NESDIS)

Updates to most datasets on our ftp site will be delayed until later this week.

$\underline{D O C}>\underline{\text { NOAA }}>\underline{\text { NESDIS }}>\underline{\text { NCDC }} \quad$ Search Field: $\quad$ Search NCDC

Climate Monitoring | Temperature and Precipitation | Drought Monitoring | NADM | Help

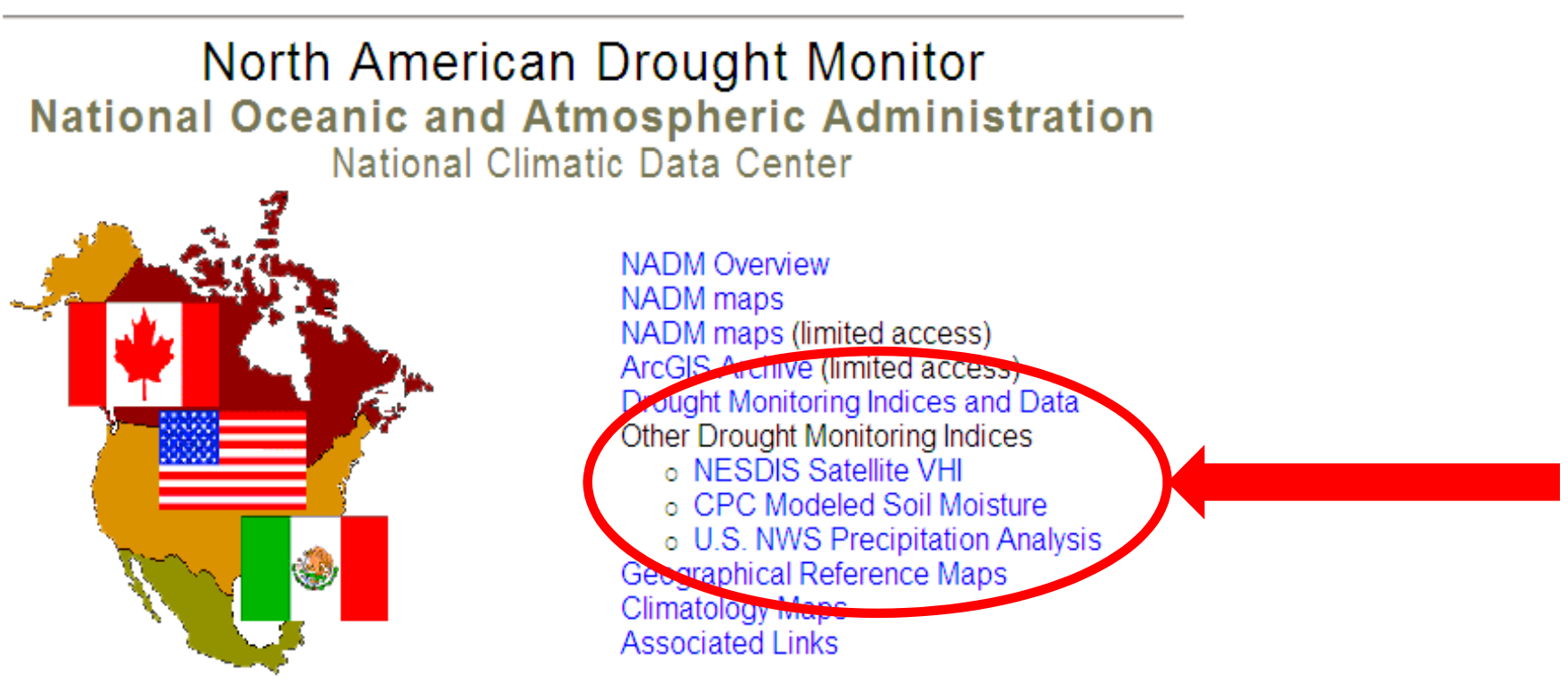

\section{North American Drought Monitor Overview}

The North America Drought Monitor (NADM) is a cooperative effort between drought experts in Canada, Mexico and the United States to monitor drought across the continent on an ongoing basis. The program was initiated at a three-day workshop in late April 2002 and is part of a larger effort to improve the monitoring of climate extremes on the continent. The NADM (Lawrimore et al. 2002) is based on the highly successful U.S. Drought Monitor (USDM), and as such, is being developed to provide an ongoing comprehensive and integrated assessment of drought throughout all three countries.

\section{http://www.ncdc.noaa.gov/oa/climate/monitoring/drought/nadm/}

\section{Nebraska}




\section{Percent of Long-Term Average Precipitation, 1-Month \\ March 2011}

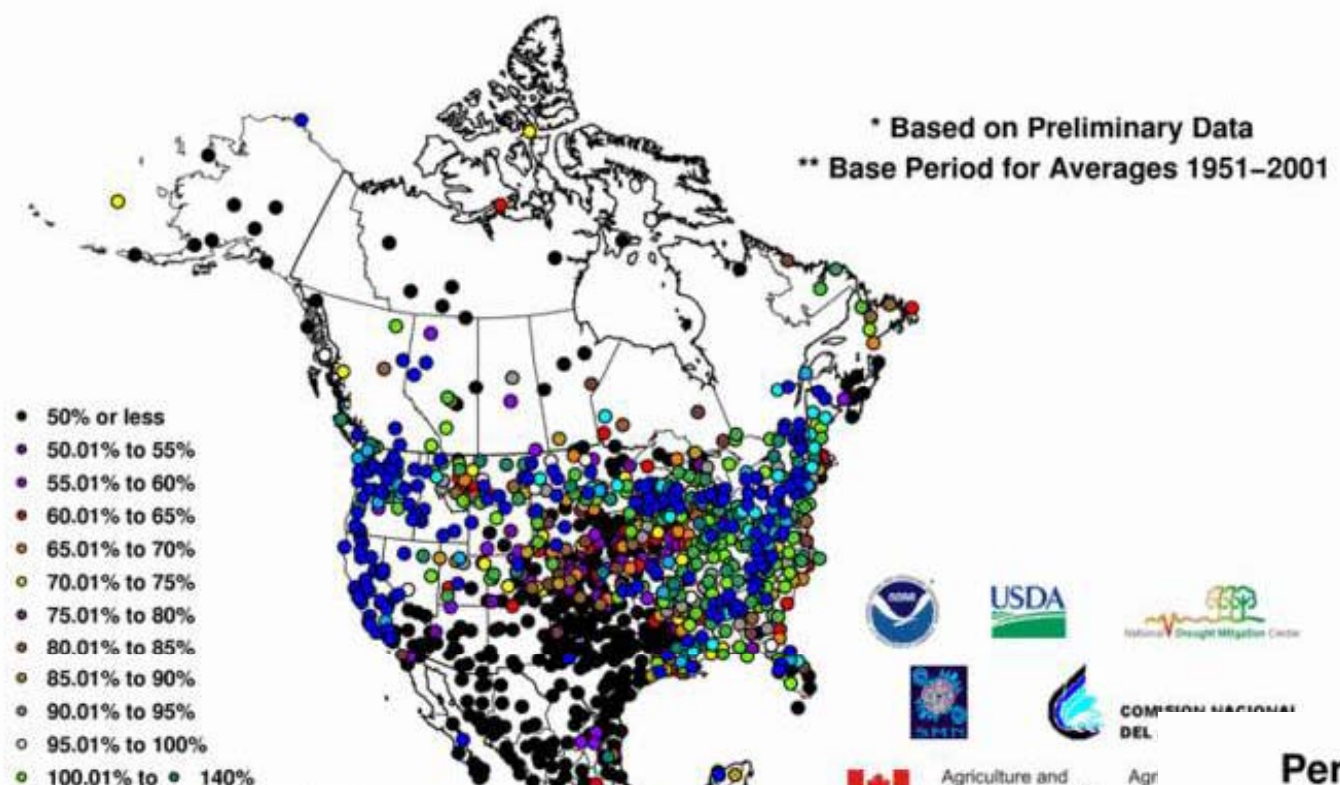

- $100.01 \%$ to $140 \%$

- $140.01 \%$ to - more than $150 \%$

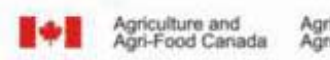
I*】 Environmont Environn

Percent of Long-Term Average Precipitation, 1-Month March 2011

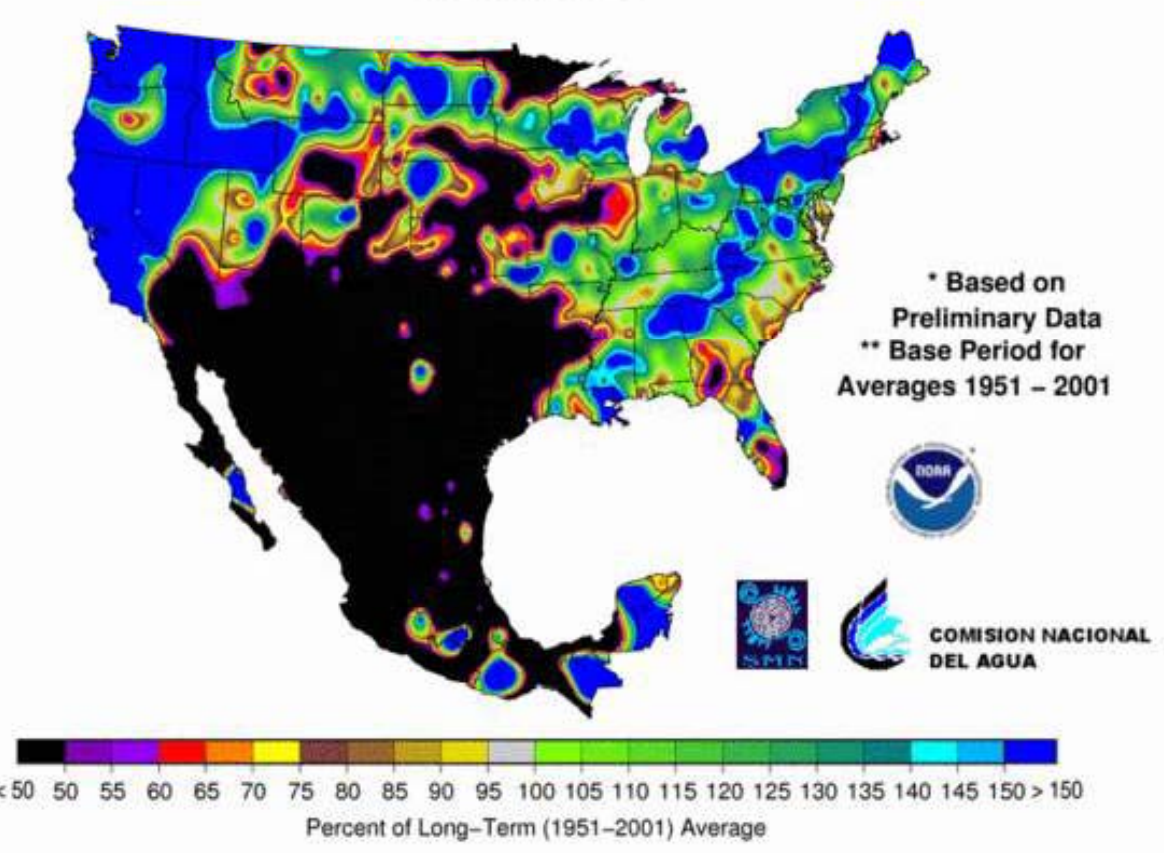




\section{6-Month Standardized Precipitation Index \\ October 2010 - March 2011}

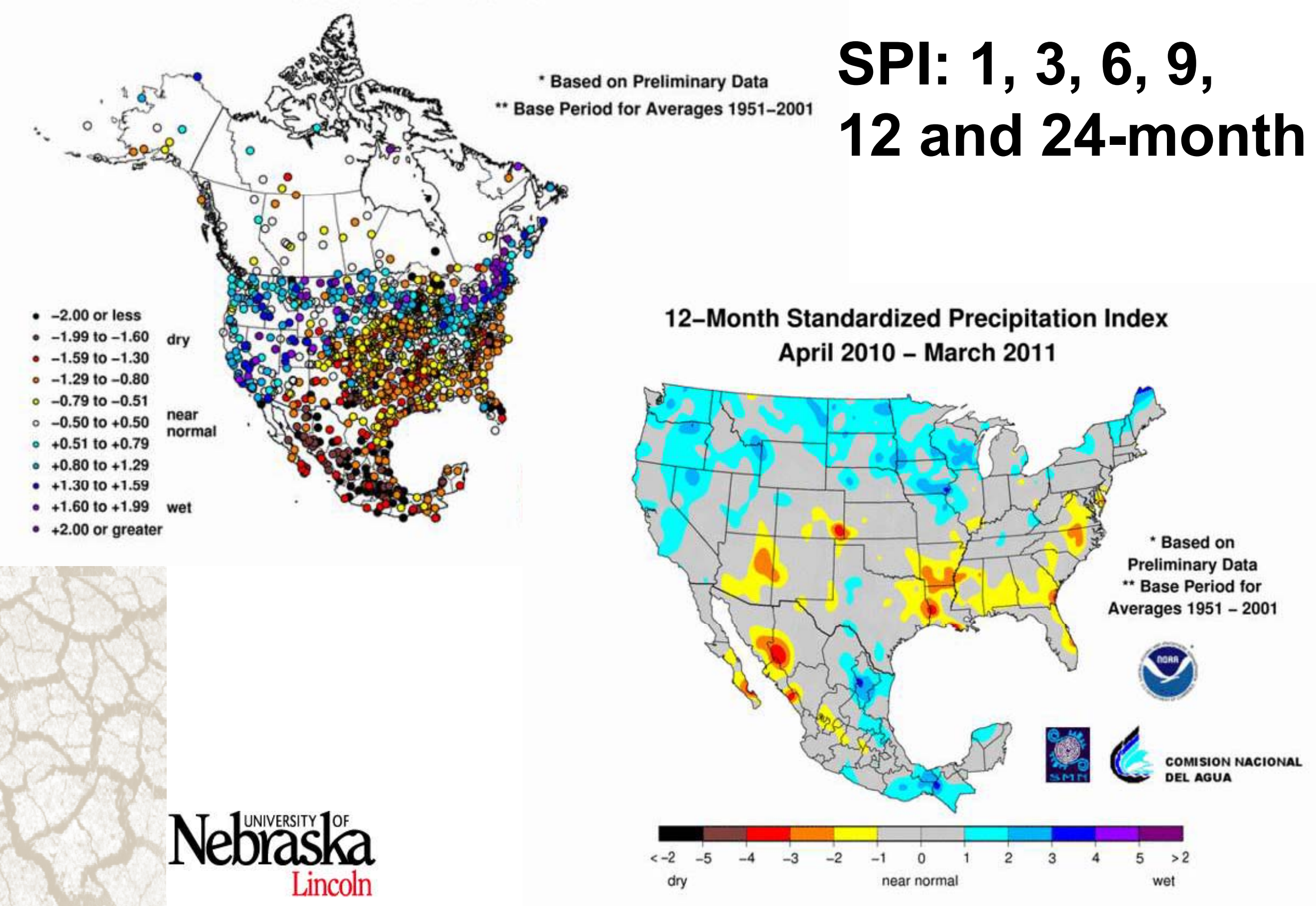




\section{Palmer Drought Index}

March 2011

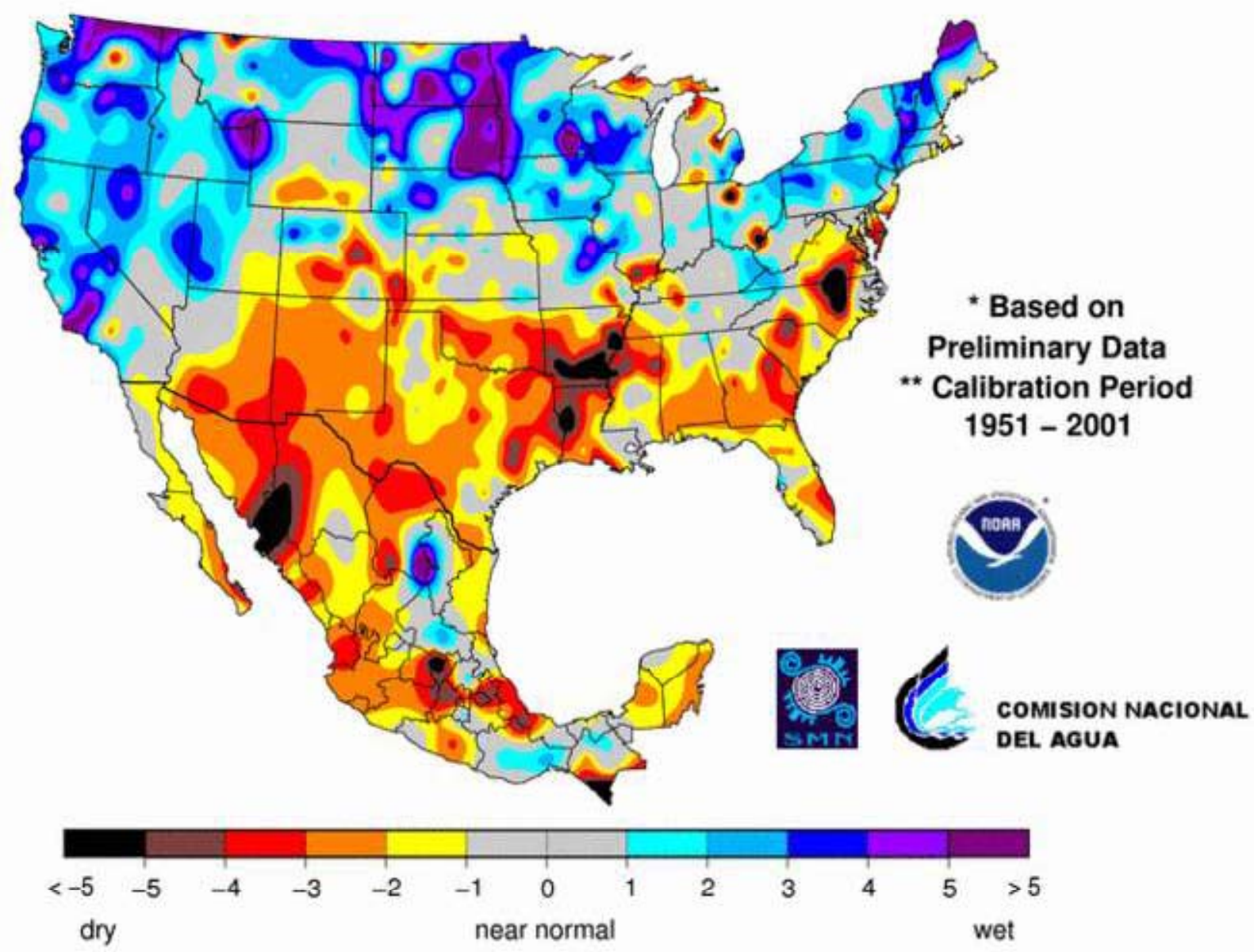

- PDSI

- PDHI

- Z 
Calculated Soil Maisture Ranking Percentile MAR 2011

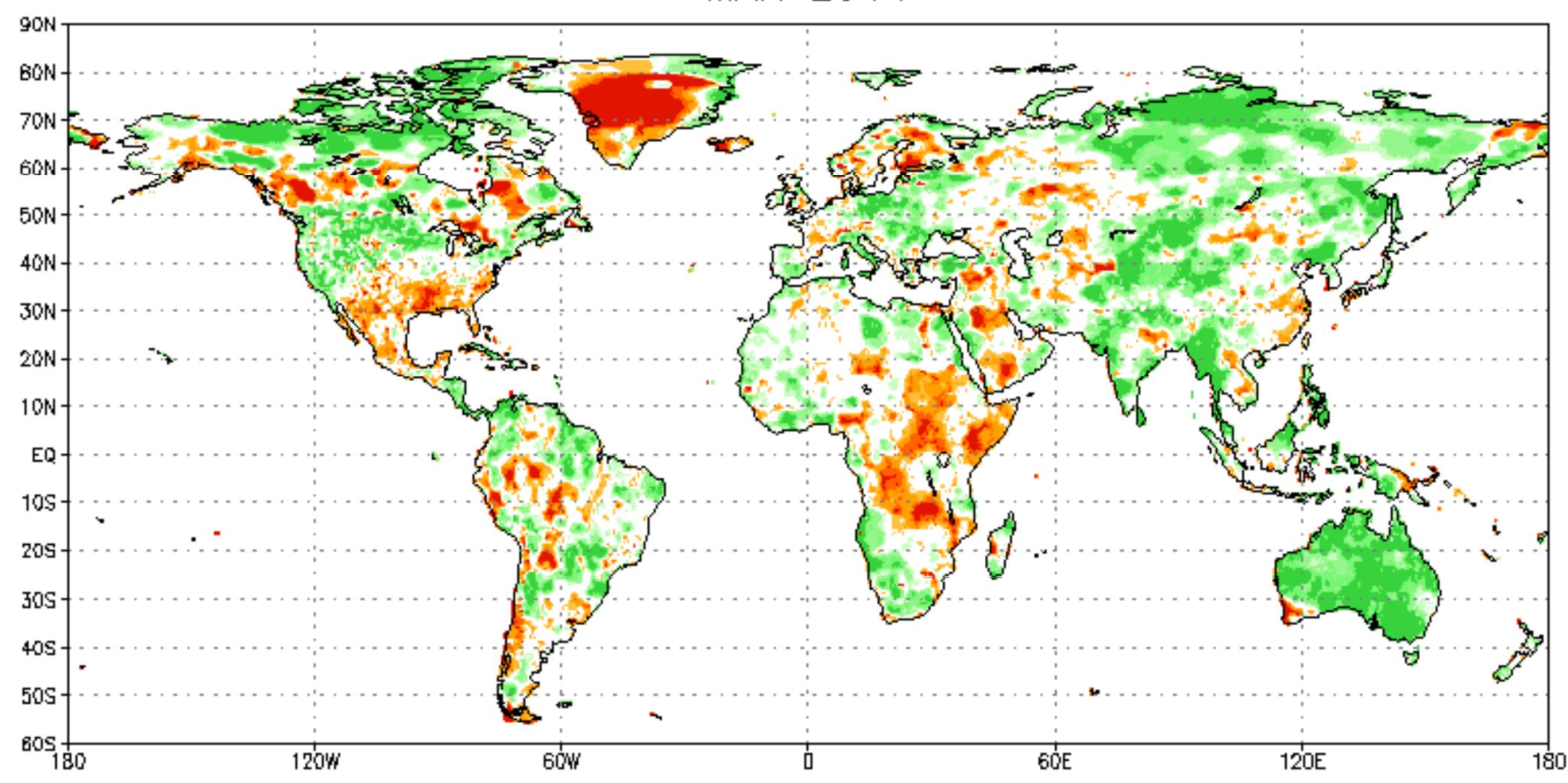

Nebraska 
Ensemble-Mean - Current Total Column Soil Moisture Anomaly (mm) NCEP NLDAS Products

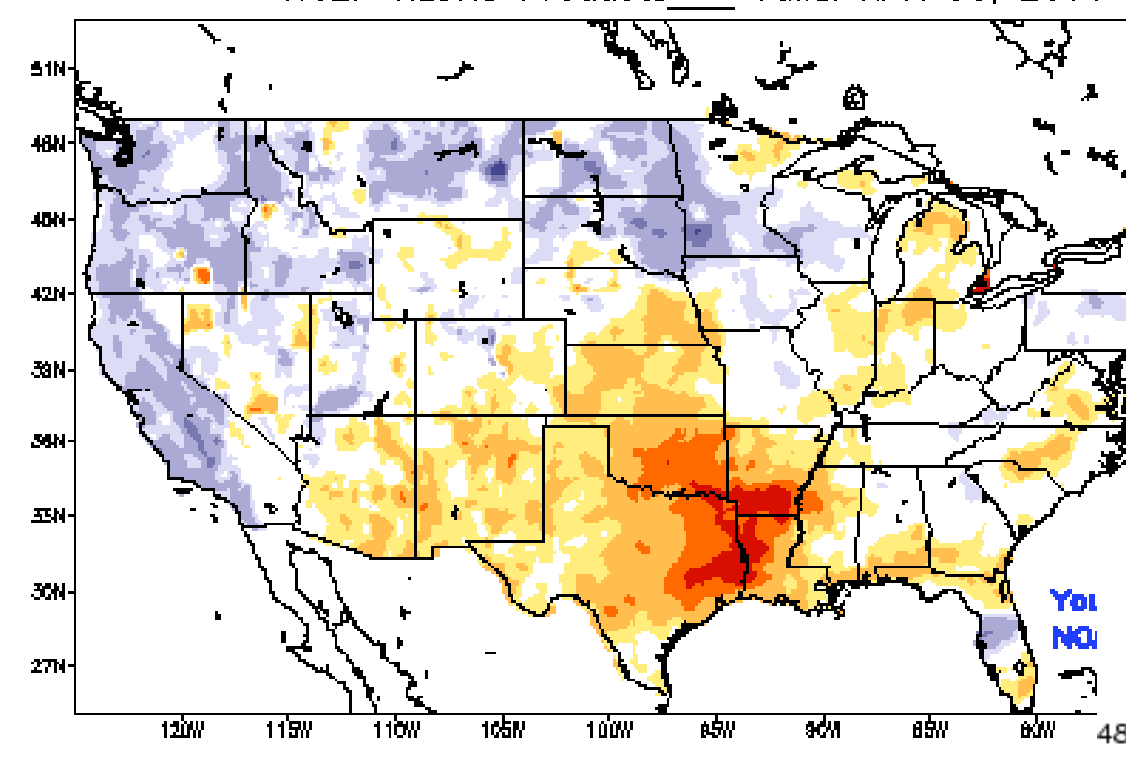

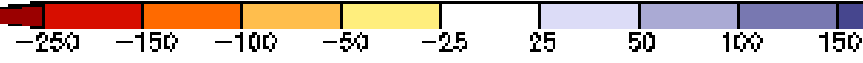

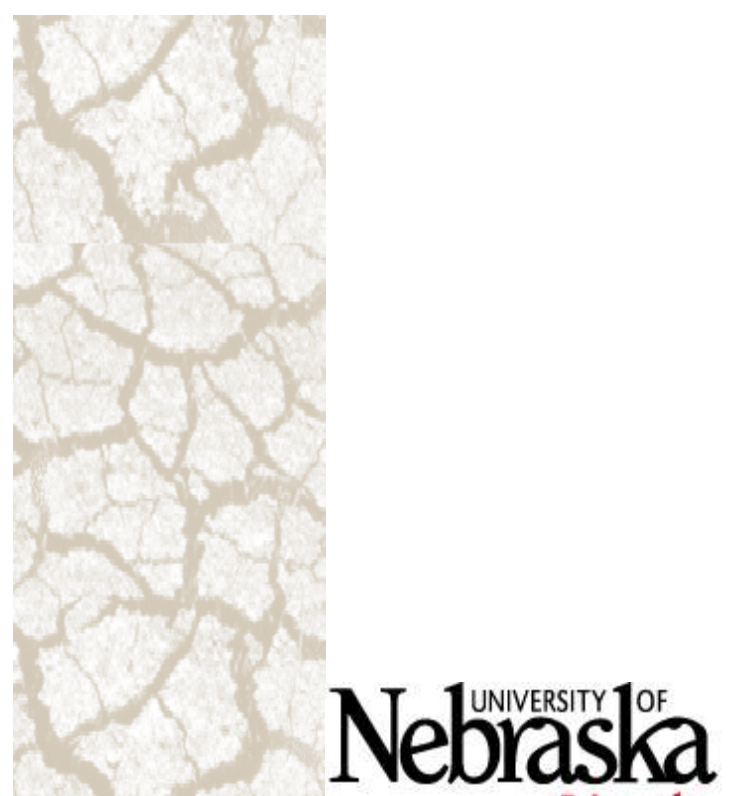

$44^{\circ}$

Lincoln

VIC Soil Moisture Percentiles (wrt/ 1916-2004) 20110407

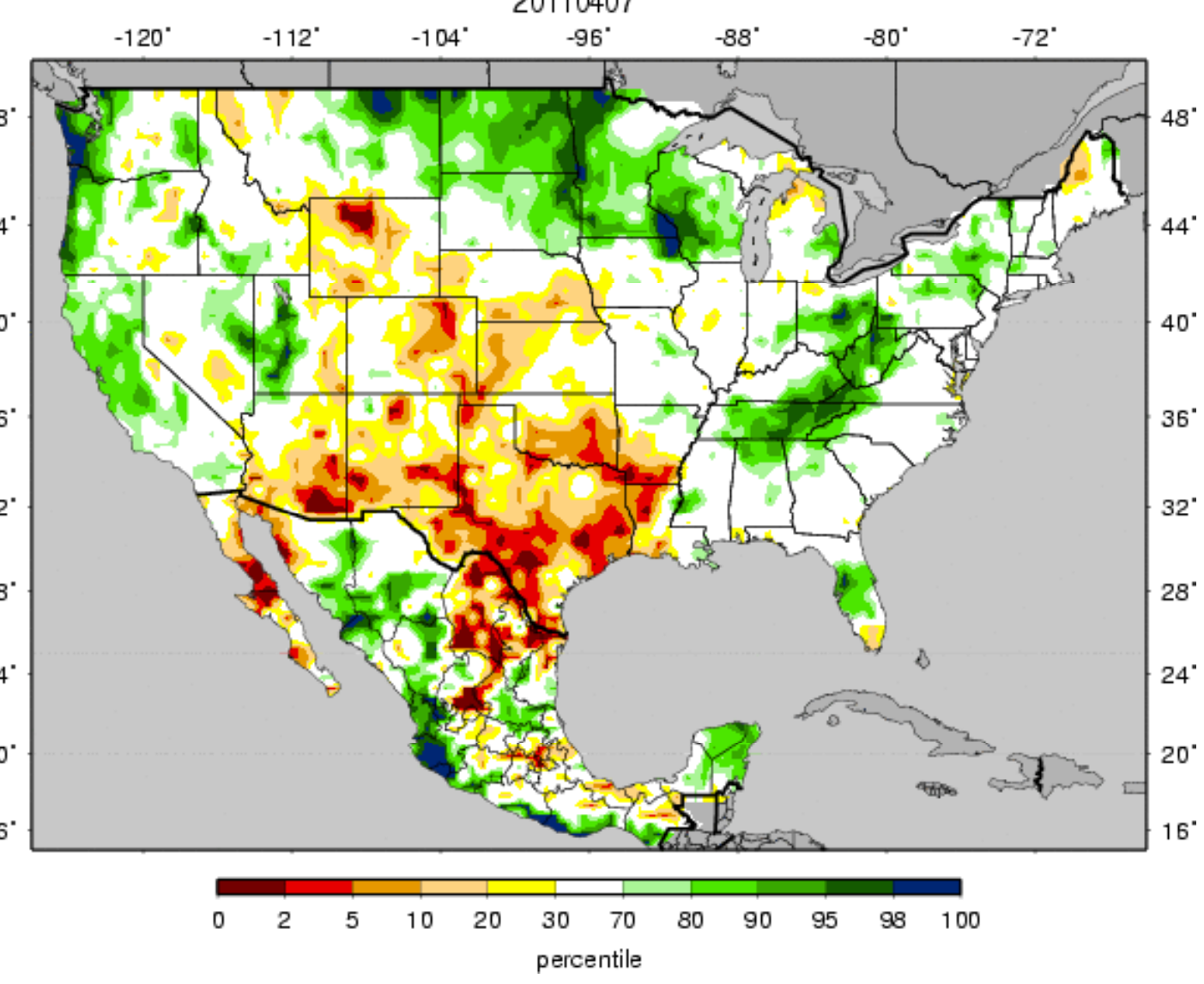




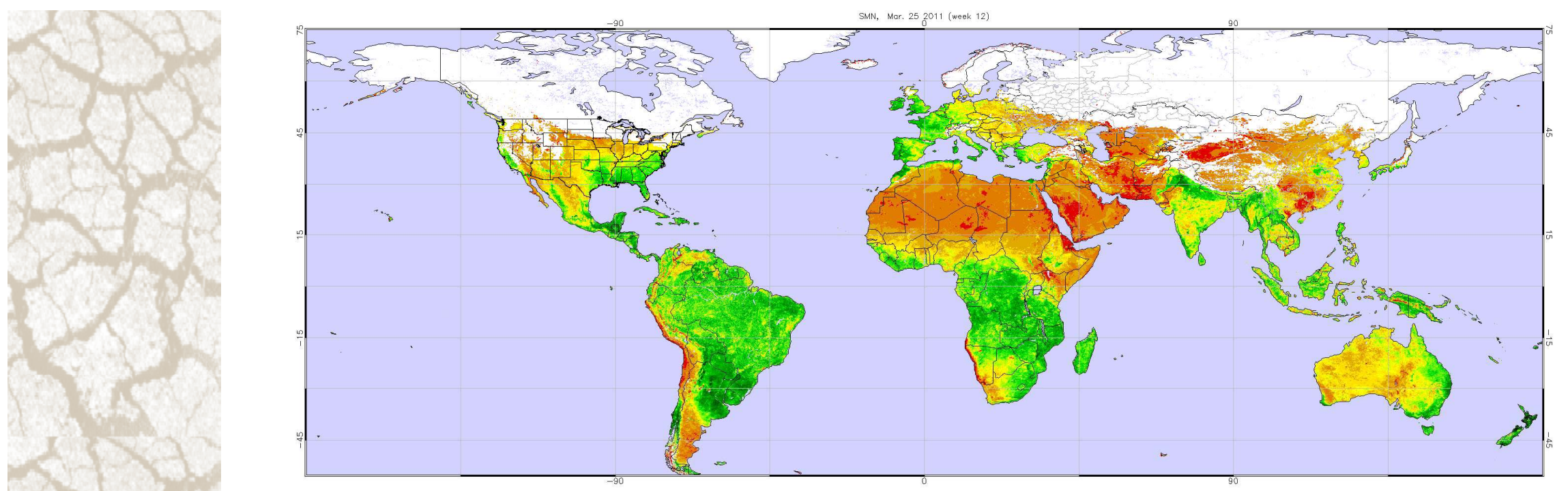

\section{Role of Remote} Sensing?

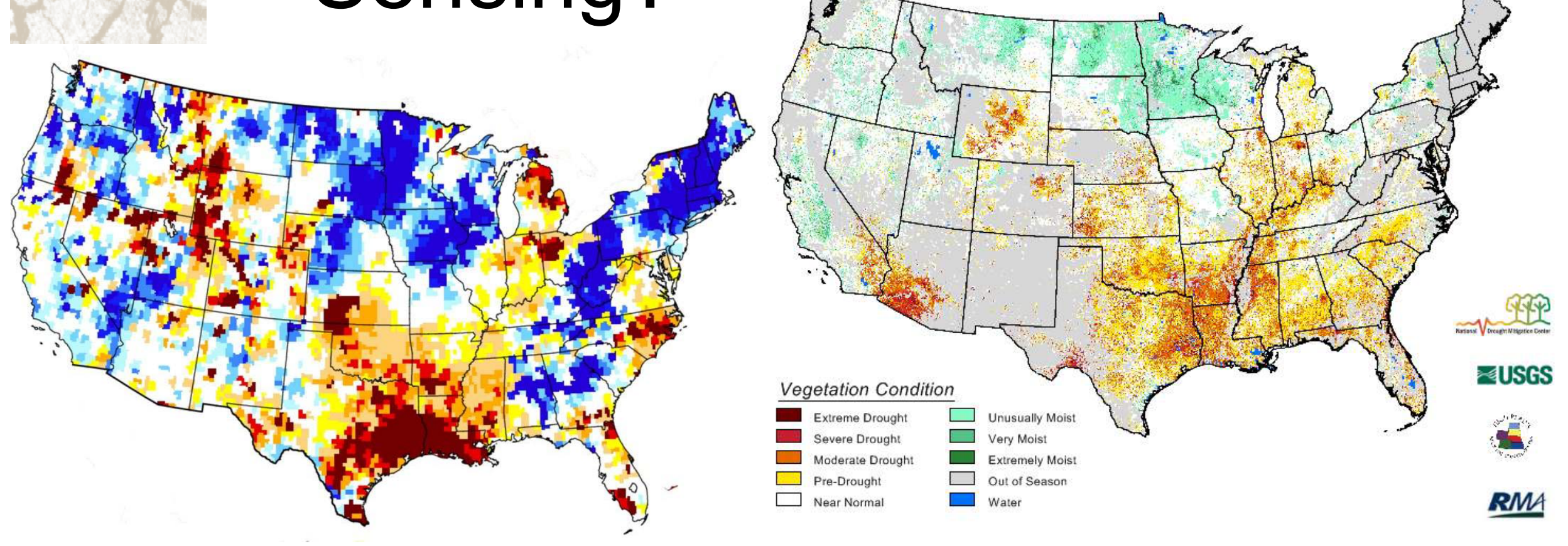




\section{Any Questions ?}

\section{Mark Svoboda \\ National Drought Mitigation Center \\ 402-472-8238 \\ msvoboda2@unl.edu}

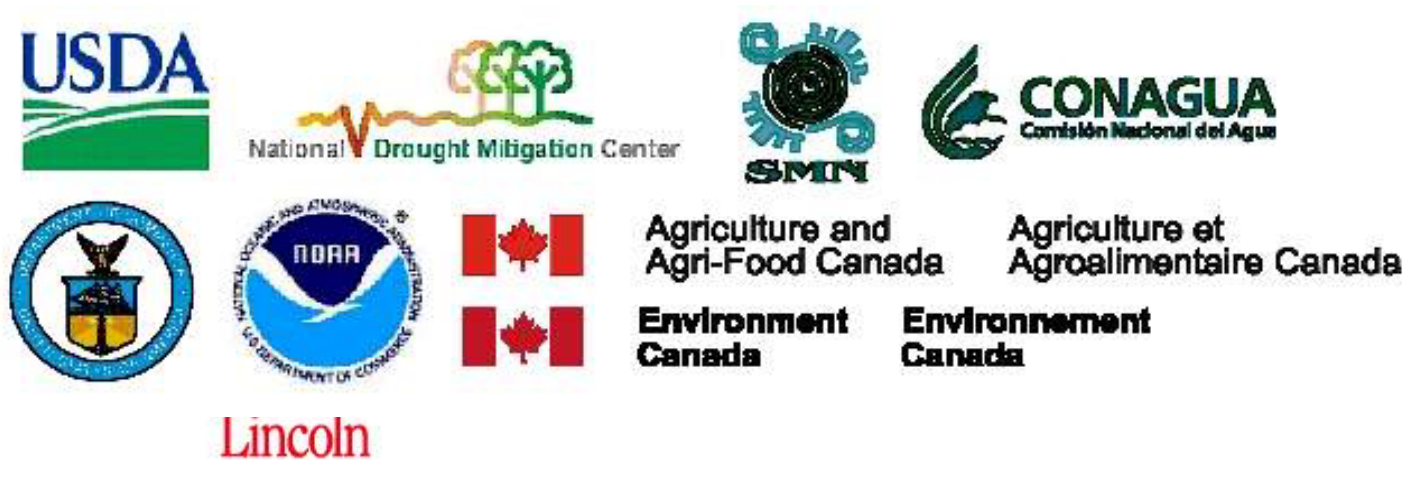


Nebraska

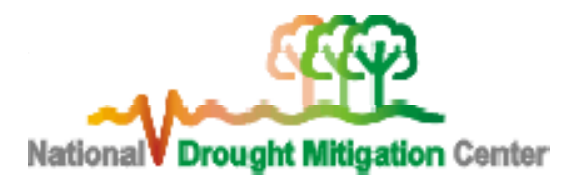


Nebraska

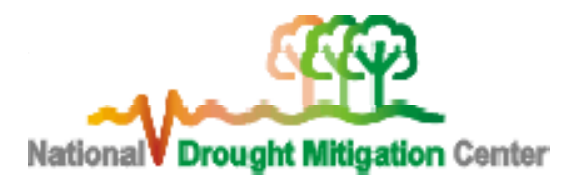

